\title{
Validation of the demographic Fetal Medicine Foundation Risk Calculator in predicting singleton antepartum stillbirth - A retrospective case-control study
}

\author{
Dana Muin ${ }^{1}$, Karin Windsperger ${ }^{1}$, Nadia Attia ${ }^{1}$, and Herbert Kiss ${ }^{1}$ \\ ${ }^{1}$ Medical University of Vienna Department of Obstetrics and Gynaecology
}

November 27, 2020

\begin{abstract}
Objectives: To externally validate the demographic setting of the online Fetal Medicine Foundation (FMF) Stillbirth Risk Calculator based upon maternal medical and obstetric history in a case-matched cohort. Design: Retrospective case-control study Setting: Tertiary referral hospital Population: 144 fetuses after singleton intrauterine fetal death (IUFD) and a matched control group of 247 singleton live births between 2003 and 2019 Methods: Nonparametric receiver operating characteristics (ROC) analysis was performed to predict the prognostic power of the risk score and to generate a cut-off value to discriminate best between the events of stillbirth versus live birth. Main Outcome Measures: FMF Stillbirth risk score Results: The IUFD cohort conveyed a significantly higher overall risk assessment with a median FMF Stillbirth risk score of 0.45\% (0.19-5.70\%) compared to live births $[0.23 \%(0.18-1.30 \%) ; \mathrm{p}<0.001]$. Demographic factors mainly contributing to the increased risk were BMI ( $p=0.002)$, smoking $(\mathrm{p}<0.001)$, chronic hypertension $(\mathrm{p}=0.015)$, APS $(\mathrm{p}=0.017)$, type 2 diabetes $(\mathrm{p}<0.001)$ and need for insulin $(\mathrm{p}<0.001)$. ROC analysis to evaluate the discriminative ability of the FMF Stillbirth Risk Calculator showed an area under the curve (AUC) of 0.72 (95\% CI 0.67-0.78; p<0.001). The FMF Stillbirth risk score at a cut-off level of $0.34 \%$ (OR 6.22; $95 \%$ CI 3.91-9.89; $\mathrm{p}<0.001$ ) yielded a specificity of $82 \%$ and a sensitivity of $58 \%$ in predicting singleton antepartum stillbirths. Conclusion: The FMF Stillbirth Risk Calculator achieved a similar performance in our cohort of women as in the reference group.
\end{abstract}

Validation of the demographic Fetal Medicine FoundationRisk Calculator in predicting singleton antepartum stillbirth - A retrospective case-control study

Dana A. Muin ${ }^{1 *}$, Karin Windsperger ${ }^{1}$, Nadia Attia ${ }^{1}$, Herbert Kiss ${ }^{1}$

${ }^{1}$ Department of Obstetrics and Gynaecology, Division of feto-maternal Medicine, Medical University of Vienna, Waehringer Guertel 18-20, 1090 Vienna, Austria

\section{*Corresponding Author:}

Dana Anaïs Muin, MD, MSc, MRes Medical University of Vienna Department of Obstetrics and Gynaecology, Division of feto-maternal Medicine Waehringer Guertel 18-20, 1090 Vienna, Austria Tel: +43 140400 28220, Fax: +4314040028620E-mail address:dana.muin@meduniwien.ac.at

ORCID iD: 000-0001-8981-4817

Running title: Validation of the demographic FMF Risk Calculator

\section{ABSTRACT}

Objectives: To externally validate the demographic setting of the online Fetal Medicine Foundation (FMF ) Stillbirth Risk Calculator based upon maternal medical and obstetric history in a case-matched cohort. 
Design: Retrospective case-control study

Setting: Tertiary referral hospital

Population: 144 fetuses after singleton intrauterine fetal death (IUFD) and a matched control group of 247 singleton live births between 2003 and 2019

Methods: Nonparametric receiver operating characteristics (ROC) analysis was performed to predict the prognostic power of the risk score and to generate a cut-off value to discriminate best between the events of stillbirth versus live birth.

Main Outcome Measures: FMF Stillbirth risk score

Results: The IUFD cohort conveyed a significantly higher overall risk assessment with a median FMF Stillbirth risk score of $0.45 \%(0.19-5.70 \%)$ compared to live births $[0.23 \%(0.18-1.30 \%) ; p<0.001]$. Demographic factors mainly contributing to the increased risk were BMI $(p=0.002)$, smoking $(p<0.001)$, chronic hypertension $(p=0.015)$, APS $(p=0.017)$, type 2 diabetes $(p<0.001)$ and need for insulin $(p<0.001)$. ROC analysis to evaluate the discriminative ability of the FMF Stillbirth Risk Calculator showed an area under the curve (AUC) of $0.72(95 \%$ CI $0.67-0.78 ; p<0.001)$. The FMF Stillbirth risk score at a cut-off level of $0.34 \%$ (OR $6.22 ; 95 \%$ CI $3.91-9.89 ; p<0.001$ ) yielded a specificity of $82 \%$ and a sensitivity of $58 \%$ in predicting singleton antepartum stillbirths.

Conclusion: The FMF Stillbirth Risk Calculator achieved a similar performance in our cohort of women as in the reference group.

Funding: This research did not receive any specific grant from funding agencies in the public, commercial, or not-for-profit sectors.

Keywords: Stillbirth; pregnancy outcome; high-risk pregnancy; epidemiology, risk prediction; validation study.

Tweetable Abstract The demographic setting of the onlineFMF Stillbirth Risk Calculator helps to identify women at high risk for antepartum \#stillbirth based upon their maternal and obstetric history \#SavingBabies@BJOGTweets @DrDana_Muin @MedUni_Wien

\section{MANUSCRIPT \\ INTRODUCTION}

Stillbirth is a devastating event and yet it harbours an up to 22 -fold recurrence risk in future pregnancies. ${ }^{1,2}$ With the aim to reduce the annual stillbirth rate worldwide, the ability to predict the likelihood of such event by accurate risk stratification may prompt both parents and clinicians to embark upon a suitable and targeted antenatal surveillance program.

Prediction models for stillbirths are most commonly defined as "models, scores or clinical decision tools" which aid in estimating the risk of stillbirth in a pregnant woman based upon certain variables. ${ }^{3}$ In a recent review, the most commonly used variables in prediction models for stillbirths have been identified to be maternal age, body mass index (BMI) and maternal diabetes, yet strongest evidence of association with stillbirth was for nulliparity, pre-existing hypertension and maternal obesity. ${ }^{4}$ As about 11.2 to $64.9 \%$ of stillbirths in high income countries are due to placental dysfunction, ${ }^{5}$ a triad of the latter factors is most likely contributing to such. Whilst the pathomechanisms of the individual risk denominators might work differently on the axis leading to fetal death, the synthesis of these variables into a prediction model is helpful for early recognition and intervention to prevent adverse perinatal outcome. To date, 69 prediction models for stillbirths have been described in literature. ${ }^{3}$

By this study we aim to apply the demographic model of the Fetal Medicine Foundation (FMF) Stillbirth Risk Calculator ${ }^{6}$ based upon maternal characteristics (weight, ethnicity and smoking), medical history [diabetes, chronic hypertension, systemic lupus erythematosus (SLE) and anti-phospholipid syndrome (APS)] and 
obstetric history (parity, stillbirth and/or preeclampsia in previous pregnancies) ${ }^{7}$ in our single-centre cohort of intrauterine fetal deaths (IUFD) and matched live births as an independent dataset for external validation of this prediction tool.

\section{METHODS}

\section{Study design and data collection}

We retrospectively reviewed all cases of singleton antepartum stillbirths and live births which were delivered at our tertiary referral centre between January 2003 and December 2019. IUFD cases, which fulfilled the study's inclusion and exclusion criteria, were matched with singleton live births for maternal age, gravidity, parity, gestational age at delivery and fetal sex (Appendix S1 ). Inclusion criteria for antepartum stillbirths were singleton IUFDs above $21^{+0}$ gestational weeks with documented fetal and maternal characteristics either at antenatal booking or at time of delivery. Exclusion criteria for IUFDs were multiple pregnancies, medical or surgical terminations of pregnancies, perinatal fetal deaths and cases with missing fetal and maternal demographics and medical history. Causes of death in IUFDs was defined as the "initial, demonstrable pathophysiological entity initiating the chain of events that has irreversibly led to death" in recognition of potentially multiple competing risks and events and was categorized according to the Causes of death and associated conditions (CODAC) classification. ${ }^{8}$

\section{Predictive variables}

Maternal age was defined as age in years at the time of stillbirth and live birth, respectively. Maternal weight (in $\mathrm{kg}$ ) was obtained at first visit. BMI was grouped as underweight ([?]18.5 kg/m²), normal weight $\left(18.6-24.9 \mathrm{~kg} / \mathrm{m}^{2}\right)$, pre-obesity $\left(25-29.9 \mathrm{~kg} / \mathrm{m}^{2}\right)$, Obesity Class I $\left(30-34.9 \mathrm{~kg} / \mathrm{m}^{2}\right)$, Obesity Class II (35-39.9 $\left.\mathrm{kg} / \mathrm{m}^{2}\right)$ and Obesity Class III ([?]40 $\left.\mathrm{kg} / \mathrm{m}^{2}\right)$. Ethnicity was self-reported by the pregnant woman at first visit and categorized into white, black, South Asian, East Asian and mixed. Smoking was defined as current smoker or non-smoker at antenatal booking or time of stillbirth. Gravidity was defined as the number of current pregnancy. Parity was defined as the number of previous deliveries (including stillbirths) above $24^{+0}$ gestational weeks or with a fetal weight of or above $500 \mathrm{~g}$. Medical and obstetric history including type 1 and 2 diabetes and respective treatment (diet, insulin, metformin), chronic hypertension, SLE, APS and previous pre-eclampsia were manually retrieved from the women's medical records.

\section{Outcome measures}

Maternal and fetal characteristics were retrieved from the electronic database ViewPoint(r) Version 5.6.28.56 (General Electric Company, Solingen, Germany). Data were transferred into a study-excel file sheet, reviewed for accuracy and made anonymous prior to analyses. Demographic variables from the study-file sheet were then manually typed into the $F M F$ Stillbirth Risk Calculator, an online tool to assess the risk for stillbirth based upon either maternal history only or in combination with first and second trimester measurements (https://fetalmedicine.org/research/assess/stillbirth). ${ }^{7,} 9-18$ For the purpose of this study, we applied the "Maternal history" setting only. The individual risk score for each case was given in percent (\%) or fractions (1:x) and was transferred into the study-excel file sheet for further analyses. The FMF Stillbirth risk score directly correlates with the degree of risk assessment.

\section{Statistical analyses}

Distribution of data was analysed using the Kolmogorov-Smirnov test. Normally distributed data are expressed as mean +- standard deviation. Not normally distributed variables are expressed as median and minimum-maximum. Categorical data are given as counts (n) and percentages (\%). Continuous data were compared with paired t-test and Wilcoxon signed-rank test, respectively. Categorical data were compared with $\mathrm{Chi}^{2}$ and Fisher's Exact test, respectively, with a 99\% Confidence Interval (CI). Nonparametric receiver operating characteristics (ROC) analysis was performed to predict the prognostic power of the risk score and to generate a cut-off value that discriminated best between the event of stillbirth versus live birth. Odds ratio (OR), as measure of the relative risk of stillbirth, was estimated using logistic regression analyses with a $95 \%$ CI. All reported $p$-values are two-sided, and level of significance was set at $<0.05$. Statistical tests 
were performed and figures created by SPSS(r) Statistics Version 26.0.0.0 (IBM Corporation (c), Armonk, NY, USA).

The study was approved by the Ethics Committee of the Medical University of Vienna (EK 1759/2018) and complied with the principles outlined in the Helsinki Declaration of 1975, as revised in 2013. Patients' written consent was not required as per Austrian Federal Act concerning Protection of Personal Data (DSG 2000).

\section{RESULTS}

\section{Maternal and fetal baseline characteristics}

Baseline maternal and fetal characteristics of stillbirths $(n=144)$ and controls $(n=247)$ are shown in Table 1. Our IUFD cohort consisted of $73(50.7 \%)$ male and $71(49.3 \%)$ female fetuses between $21^{+1}$ and $41^{+3}$ gestational weeks at a median age of $31^{+4}$ gestational weeks. Median fetal weight at stillbirth was 1397 (180-4450)g. Causes of IUFD according to CODAC were congenital fetal malformations in 72 (50.0\%) cases; placental pathologies in $26(18.1 \%)$ cases; unknown despite thorough investigation or due to lack of important information in $25(17.4 \%)$ cases; fetal conditions in 9 (6.3\%) cases, umbilical cord complications in $6(4.2 \%)$ cases, maternal conditions in $4(2.8 \%)$ cases and IUFD due to infection in $2(1.4 \%)$ cases.

The matched live birth cohort consisted of 138 (55.9\%) male and 109 (44.1\%) female fetuses born alive between $21^{+1}$ and $41^{+3}$ gestational weeks at a median age of $31^{+6}$ gestational weeks. Median newborn weight at delivery was 1880 (341-4540)g with a median Apgar score of 8 points at 1 minute and 9 points at 5 and 10 minutes, respectively, with a median arterial $\mathrm{pH}$ of 7.29 (6.89-7.46). From the five matched live births under $22^{+0}$ gestational weeks, case 1 was noted to have a univentricular heart (male, birth weight 482g, Apgar 1/1/1), case 2 had a preterm delivery following premature rupture of membranes (female, 354g, Apgar 2/1/1), case 3 had bilateral kidney agenesis with oligohydramnios (male, 341g, Apgar 1/1/1), case 4 suffered amniotic infection followed by preterm delivery (male, 440g, Apgar 1/0/0) and case 5 had commissural agenesis and vermis hypoplasia (male, 541g, Apgar 2/1/1). None of those extremely premature newborns survived the first 2 weeks of life.

\section{Fetal Medicine Foundation Stillbirth risk score}

Table 1 shows the maternal demographic data that synthesize the FMF Stillbirth Risk Calculator. In our study cohort, women after stillbirth compared to matched controls were found to have a significantly higher BMI $(p=0.002)$, were more frequently nicotine consumers $(p<0.001)$ and suffered more frequently medical conditions, such as hypertension $(p=0.015)$, APS $(p=0.017)$ and diabetes $(p<0.001)$ with higher need for insulin $(p=0.006)$.

Figure 1 shows the distribution of the FMFStillbirth risk score in IUFDs and matched controls. The medianFMF Stillbirth risk score in the group of stillbirths was $0.45 \%(0.19-5.70 \%)$ [1:222 (1:526-1:17)], whilst the median risk score in the group of matched live births was $0.23 \%(0.18-1.30 \%)$ [1:435 (1:556-1:77); $p<0.001]$. Figure 2 and Figure 3 show the FMF Stillbirth risk scores in stillborn fetuses per cause of death with and without outliers, respectively.

To evaluate the discriminative power of the FMF Stillbirth Risk Calculator we performed a ROC analysis and calculated an area under the curve (AUC) of $0.72(95 \%$ CI $0.67-0.78 ; p<0.001)$ to predict antepartum stillbirth in the total cohort (Figure 4 ). Also after exclusion of all stillborn fetuses with congenital anomalies, the AUC was $0.74(95 \%$ CI $0.67-0.82 ; p<0.001)$. Assessing stillborn fetuses of unknown cause, AUC was $0.83(95 \%$ CI $0.72-0.93 ; p<0.001)$. Assessing stillborn fetuses due to placental dysfunction, the AUC was $0.64(95 \%$ CI $0.50-0.78 ; p=0.053)$.

Univariate binary logistic regression to examine the FMFStillbirth Risk Calculator's predictive ability resulted in an OR of $6.22(95 \%$ CI $3.91-9.89 ; p<0.001)$ at an optimalFMF Stillbirth risk score cut-off of [?] $0.34 \%$ for predicting stillbirth with a specificity of $82 \%$ and a sensitivity of $58 \%$ in the total cohort of IUFDs. 


\section{DISCUSSION}

\section{Main findings}

In this study, we applied the FMF Stillbirth Risk Calculator based upon demographic characteristics in an independent case-matched dataset. We found that maternal, medical and obstetric history yield a high specificity and satisfactory sensitivity in discriminating between the outcomes live birth versus stillbirth. In acknowledgment of the challenge to predict adverse events in the presence of competing risks at later stages in pregnancy, the demographic maternal patterns, which may remain unaltered most often during pregnancy, may assist clinical risk assessment as early as at antenatal booking.

\section{Strengths and limitations}

The strengths of this study are first, the strict inclusion and exclusion criteria in the cohort of singleton antepartum stillbirths, each of which has been subject to extensive post-mortem investigations in order to define the cause of death. Our institutional database on antepartum stillbirths includes reliable and accurate data, which are annually checked and updated. This ascertains a continuous degree of quality. The singlecentre setting of the study also reduces bias and heterogeneity in reporting pathology findings and collection of pregnancy data.

However, we acknowledge the relatively small sample size due to the study's design and retrospective setting. Also, maternal demographic parameters were self-reported by the woman at time of antenatal booking or delivery and therefore subject to recall bias which we cannot control for.

\section{Interpretation}

Despite these limitations, this study is the first to independently assess and validate the FMF Stillbirth Risk Calculator in a cohort restricted to IUFDs and matched live births. In our dataset, theFMF Stillbirth Risk Calculator achieved a similar performance as in the reference group. ${ }^{7}$

Two characteristics in our cohort are of note: First, we included both stillborn and live born fetuses with congenital anomalies. Despite this variation to the reference cohort, the prediction model still remained accurate with and without the consideration of congenital anomalies in our population. A recent secondary analysis of a case-control study confirmed that among stillbirths, $23.4 \%$ had one or more major anomalies compared to $4.3 \%$ of live births. ${ }^{19}$ Yet, taking these data together, it seems justified to prospectively use this model for risk stratification in stillbirth as early as at preconception, thus ahead of any eventual detection of fetal congenital anomalies later in pregnancy. Also, we considered all IUFD cases from gestational week $21^{+0}$ onwards. One reason for this is the international heterogeneity in definitions of stillbirth by gestational weeks and to address those who define fetal death as early as of week $20 .^{3}$

There is a robust body of evidence for the link of nulliparity, pre-existing hypertension and increased maternal BMI to antepartum stillbirth. ${ }^{4}$ The demographic findings from our cohort support these data, as all but nulliparity were significantly more prevalent in women affected by fetal death.

\section{Clinical and research implications}

Townsend et al. have proposed that in the future development of a robust risk prediction tool for stillbirth the following candidate variables should be incorporated: maternal age, BMI, parity, pre-existing hypertension, diabetes, previous stillbirth, nicotine consumption, uterine artery Doppler, pregnancy-associated plasma protein PAPP-A and placental growth factor PlGF. ${ }^{4}$ The merit of such clinical model would be twofold: primarily, the accurate discrimination of high- from low risk pregnant women, and secondarily, recognizing the variables that may require early enough alteration if they are modifiable. Whilst maternal age, parity, previous stillbirths and biomarkers cannot be adapted by intervention, maternal weight, hypertension, type 2 diabetes and nicotine consumption can be improved through life style modifications. To extrapolate this concept to the demographic model of the FMF Stillbirth Risk Calculator, only four variables may be potential subjects to change and therefore possible risk reduction (weight, smoking, diabetes, hypertension). As with many other risk-adjustment models, however, social and behavioural variables, such as domestic 
abuse, stress, employment and deprivation are hard to capture and should be further considered within a population-based conceptual framework. ${ }^{20}$ Additional research into prediction models may objectify the true preventability of stillbirth by adaption of modifiable risk factors in the future.

\section{CONCLUSION}

Raising awareness for maternal epidemiological risk factors is an important measure in antenatal care. The demographic setting of the online Fetal Medicine Foundation Stillbirth Risk Calculator may be a useful tool to identify women at risk for antepartum stillbirth. Its implementation into clinical practice as early as in the preconception period might support obstetrical counselling and prompt timely intervention to prevent adverse neonatal outcome. Further validation in prospective and larger cohort studies is needed.

\section{Conflict of Interest}

None declared. Completed disclosure of interests form available to view online as supporting information.

\section{Contribution to authorship}

All authors contributed to the conception, planning and carrying out of the research. DAM conceived and designed the study and wrote the first draft of this paper. KW and NA helped with acquisition of data. DAM and KW conducted statistical analyses. KW, NA and HK helped with design and conception. All authors contributed to critically revising the paper for important intellectual content and approved the final version to be published. All authors accept responsibility for the article as published.

\section{Details of ethical approval}

The study was approved by the Ethics Committee of the Medical University of Vienna (EK 1759/2018) and complied with the principles outlined in the Helsinki Declaration of 1975, as revised in 2013. Patients' written consent was not required as per Austrian Federal Act concerning Protection of Personal Data (DSG 2000).

\section{Funding}

None.

\section{Acknowledgments}

DAM is thankful to Professor DDr. J.C. Huber for his enduring support.

\section{SUPPORTING INFORMATION}

Appendix S1. Flow diagram on the selection of fetal death cases and matched controls

\section{TABLES AND FIGURES}

Table 1. Maternal and fetal characteristics of the study cohort

Figure 1. Distribution of the Fetal Medicine Foundation Stillbirth risk score in fetal death cases and matched controls

Figure 2. Box-plot analysis including outliers showing Fetal Medicine Foundation Stillbirth risk score in stillborns per cause of fetal death (CODAC Classification). Red line signifies of optimal cut-off point at $0.34 \%$.

Figure 3. Box-plot analysis excluding outliers showing Fetal Medicine Foundation Stillbirth risk score in stillborns per cause of fetal death (CODAC Classification). Red line signifies of optimal cut-off point at $0.34 \%$.

Figure 4. Receiver operating characteristic (ROC) curve for the Fetal Medicine Foundation Stillbirth risk score to predict stillbirth, area under the ROC curve (AUC) $0.72(95 \%$ CI $0.67-0.78 ; p<0.001)$

\section{References}


1. Bhattacharya S, Prescott GJ, Black M, Shetty A. Recurrence risk of stillbirth in a second pregnancy. BJOG : an international journal of obstetrics and gynaecology. 2010 Sep;117(10):1243-7.

2. Lamont K, Scott NW, Jones GT, Bhattacharya S. Risk of recurrent stillbirth: systematic review and meta-analysis. BMJ (Clinical research ed). 2015 Jun 24;350:h3080.

3. Townsend R, Manji A, Allotey J, Heazell A, Jorgensen L, Magee LA, et al. Can risk prediction models help us individualise stillbirth prevention? A systematic review and critical appraisal of published risk models. BJOG : an international journal of obstetrics and gynaecology. 2020 Sep 7.

4. Townsend R, Sileo FG, Allotey J, Dodds J, Heazell A, Jorgensen L, et al. Prediction of stillbirth: an umbrella review of evaluation of prognostic variables. BJOG : an international journal of obstetrics and gynaecology. 2020 Sep 15.

5. Ptacek I, Sebire NJ, Man JA, Brownbill P, Heazell AE. Systematic review of placental pathology reported in association with stillbirth. Placenta. 2014 Aug;35(8):552-62.

6. Fetal Medicine Foundation. Risk Assessment - Risk for stillbirth. 2018 [cited; Available from: https://fetalmedicine.org/research/assess/stillbirth

7. Yerlikaya G, Akolekar R, McPherson K, Syngelaki A, Nicolaides KH. Prediction of stillbirth from maternal demographic and pregnancy characteristics. Ultrasound in obstetrics \& gynecology : the official journal of the International Society of Ultrasound in Obstetrics and Gynecology. 2016 Nov;48(5):607-12.

8. Froen JF, Pinar H, Flenady V, Bahrin S, Charles A, Chauke L, et al. Causes of death and associated conditions (Codac): a utilitarian approach to the classification of perinatal deaths. BMC pregnancy and childbirth. 2009 Jun 10;9:22.

9. Akolekar R, Bower S, Flack N, Bilardo CM, Nicolaides KH. Prediction of miscarriage and stillbirth at 11-13 weeks and the contribution of chorionic villus sampling. Prenatal diagnosis. 2011 Jan;31(1):38-45.

10. Akolekar R, Machuca M, Mendes M, Paschos V, Nicolaides KH. Prediction of stillbirth from placental growth factor at 11-13 weeks. Ultrasound in obstetrics \& gynecology : the official journal of the International Society of Ultrasound in Obstetrics and Gynecology. 2016 Nov;48(5):618-23.

11. Akolekar R, Machuca M, Mendes M, Paschos V, Nicolaides KH. Placental growth factor in prediction of stillbirths at 11-13 weeks. Ultrasound in obstetrics \& gynecology : the official journal of the International Society of Ultrasound in Obstetrics and Gynecology. 2016 Aug 26.

12. Akolekar R, Syngelaki A, Gallo DM, Poon LC, Nicolaides KH. Umbilical and fetal middle cerebral artery Doppler at 35-37 weeks' gestation in the prediction of adverse perinatal outcome. Ultrasound in obstetrics \& gynecology : the official journal of the International Society of Ultrasound in Obstetrics and Gynecology. 2015 Jul;46(1):82-92.

13. Akolekar R, Tokunaka M, Ortega N, Syngelaki A, Nicolaides KH. Prediction of stillbirth from maternal factors, fetal biometry and uterine artery Doppler at 19-24 weeks. Ultrasound in obstetrics \& gynecology : the official journal of the International Society of Ultrasound in Obstetrics and Gynecology. 2016 Nov;48(5):62430 .

14. Akolekar R, Tokunaka M, Ortega N, Syngelaki A, Nicolaides KH. Prediction of stillbirth from maternal factors, fetal biometry and uterine artery Doppler at 19-24 weeks' gestation. Ultrasound in obstetrics \& gynecology : the official journal of the International Society of Ultrasound in Obstetrics and Gynecology. 2016 Sep 07.

15. Aupont JE, Akolekar R, Illian A, Neonakis S, Nicolaides KH. Prediction of stillbirth from placental growth factor at 19-24 weeks. Ultrasound in obstetrics \& gynecology : the official journal of the International Society of Ultrasound in Obstetrics and Gynecology. 2016 Nov;48(5):631-5. 
16. Aupont JE, Akolekar R, Illian A, Neonakis S, Nicolaides KH. Prediction of stillbirth by placental growth factor at 19-24 weeks' gestation. Ultrasound in obstetrics \& gynecology : the official journal of the International Society of Ultrasound in Obstetrics and Gynecology. 2016 Aug 18.

17. Bakalis S, Akolekar R, Gallo DM, Poon LC, Nicolaides KH. Umbilical and fetal middle cerebral artery Doppler at 30-34 weeks' gestation in the prediction of adverse perinatal outcome. Ultrasound in obstetrics \& gynecology : the official journal of the International Society of Ultrasound in Obstetrics and Gynecology. 2015 Apr;45(4):409-20.

18. Chaveeva P, Carbone IF, Syngelaki A, Akolekar R, Nicolaides KH. Contribution of method of conception on pregnancy outcome after the 11-13 weeks scan. Fetal diagnosis and therapy. 2011;30(1):9-22.

19. Son SL, Allshouse AA, Page JM, Debbink MP, Pinar H, Reddy U, et al. Stillbirth and Fetal Anomalies: secondary analysis of a case-control study. BJOG : an international journal of obstetrics and gynaecology. 2020 Sep 18.

20. Heazell AEP, Budd J, Smith LK, Li M, Cronin R, Bradford B, et al. Associations between Social and Behavioural Factors and the risk of Late Stillbirth - Findings from the Midland and North of England Stillbirth Case-Control Study. BJOG : an international journal of obstetrics and gynaecology. 2020 Sep 29.

\section{Hosted file}

Table_1.pdf available at https://authorea.com/users/363757/articles/495579-validationof-the-demographic-fetal-medicine-foundation-risk-calculator-in-predicting-singletonantepartum-stillbirth-a-retrospective-case-control-study

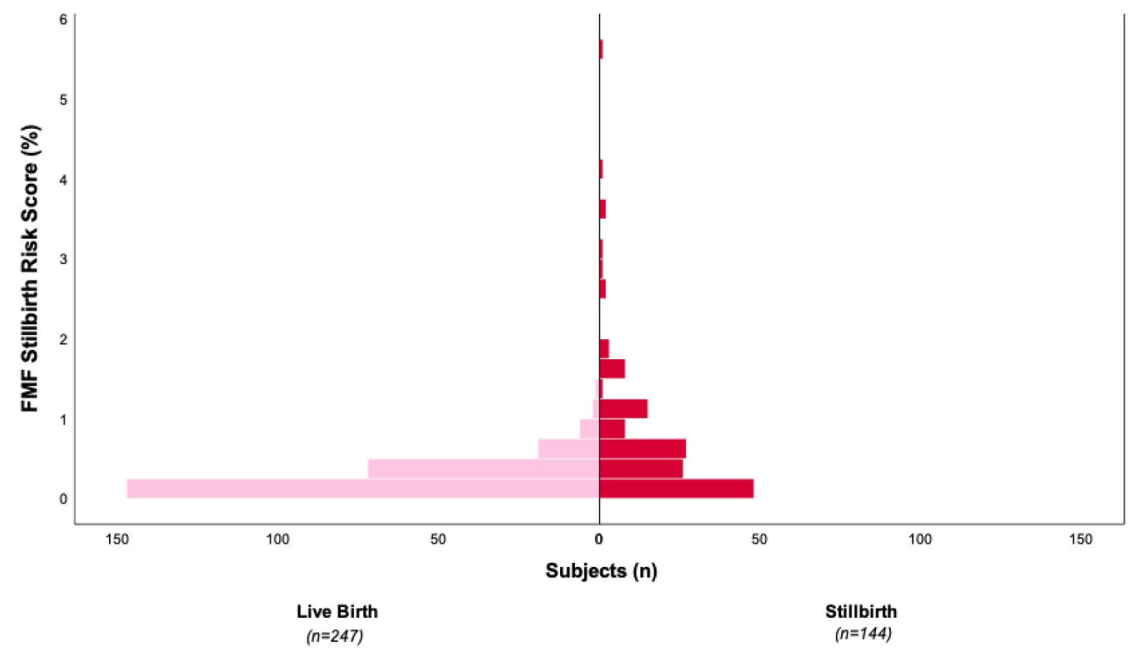



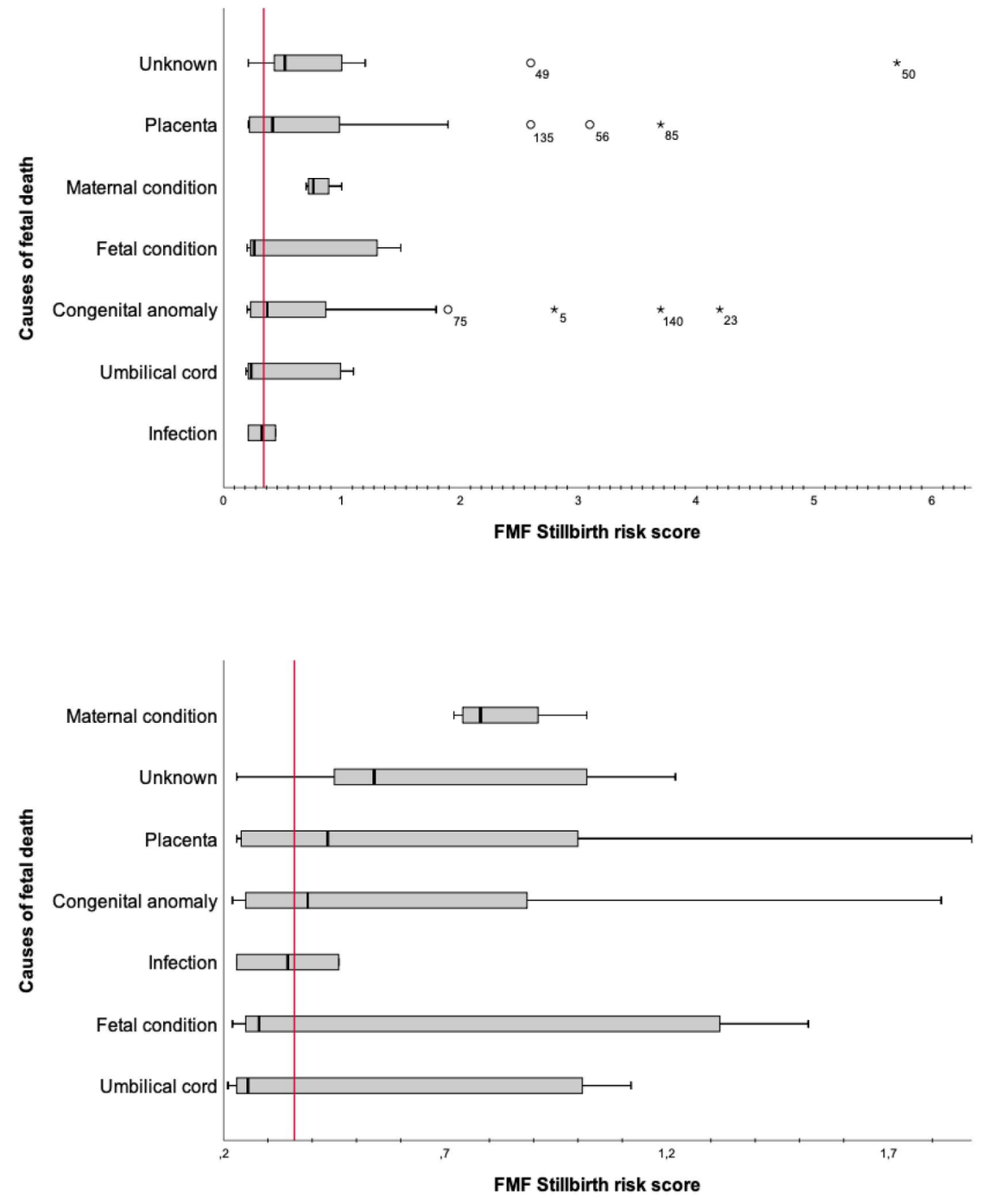


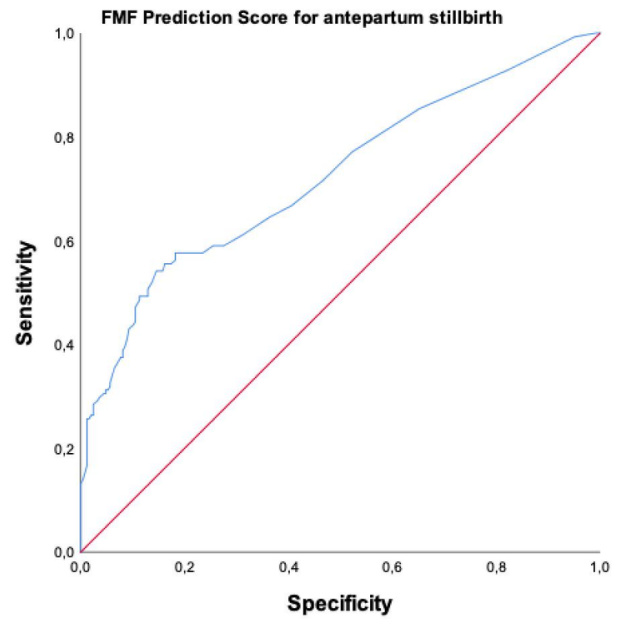

